\title{
Zebrafish and medaka: model organisms for a comparative developmental approach of brain asymmetry
}

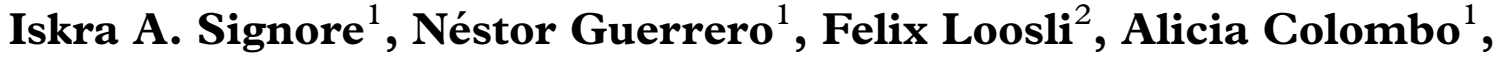 \\ Aldo Villalón ${ }^{1}$, Joachim Wittbrodt ${ }^{3}$ and Miguel L. Concha ${ }^{1, *}$ \\ ${ }^{1}$ Laboratory of Experimental Ontogeny, Nucleus of Neural Morphogenesis, \\ Anatomy and Developmental Biology Program, ICBM, Faculty of Medicine, University of Chile, \\ Independencia 1027, 8380453 Santiago, Chile \\ ${ }^{2}$ Institut für Toxikologie und Genetik, Forschungszentrum Karlsruhe, Hermann-von-Helmholtz-Platz 1, \\ 76344 Eggenstein-Leopoldshafen, Germany \\ ${ }^{3}$ Developmental Biology Programme, EMBL-Heidelberg, Meyerhofstrasse 1, 69012 Heidelberg, Germany
}

Comparison between related species is a successful approach to uncover conserved and divergent principles of development. Here, we studied the pattern of epithalamic asymmetry in zebrafish (Danio rerio) and medaka (Oryzias latipes), two related teleost species with 115-200 Myr of independent evolution. We found that these species share a strikingly conserved overall pattern of asymmetry in the parapineal-habenular-interpeduncular system. Nodal signalling exhibits comparable spatial and temporal asymmetric expressions in the presumptive epithalamus preceding the development of morphological asymmetries. Neuroanatomical asymmetries consist of left-sided asymmetric positioning and connectivity of the parapineal organ, enlargement of neuropil in the left habenula compared with the right habenula and segregation of left-right habenular efferents along the dorsoventral axis of the interpeduncular nucleus. Despite the overall conservation of asymmetry, we observed heterotopic changes in the topology of parapineal efferent connectivity, heterochronic shifts in the timing of developmental events underlying the establishment of asymmetry and divergent degrees of canalization of embryo laterality. We offer new tools for developmental time comparison among species and propose, for each of these transformations, novel hypotheses of ontogenic mechanisms that explain interspecies variations that can be tested experimentally. Together, these findings highlight the usefulness of zebrafish and medaka as comparative models to study the developmental mechanisms of epithalamic asymmetry in vertebrates.

Keywords: brain asymmetry; development; teleosts; laterality; epithalamus; heterochrony

\section{INTRODUCTION}

Asymmetry is a fundamental and conserved feature of the brain, which is thought to enhance information processing and task performance in behaviours central for species survival, such as feeding, predator detection and memory (Güntürkün et al. 2000; Rogers 2000; Pascual et al. 2004; Vallortigara \& Rogers 2005; Rogers \& Vallortigara 2008). In addition, asymmetry has been proposed as the basis of speech and other behavioural traits (Sherman et al. 1982; Rogers \& Andrew 2002; Hutsler \& Galuske 2003; Toga \& Thompson 2003) and abnormal asymmetry appears to associate with several neuropathologies including schizophrenia (Li et al. 2007), autism (Escalante-Mead et al. 2003) and neuronal degenerative diseases (Toth et al. 2004). In the last decade, experimental studies have provided valuable insights into the developmental basis of brain asymmetry. Particularly helpful have been genetic model organisms that allow a comprehensive

* Author for correspondence (mconcha@med.uchile.cl).

One contribution of 14 to a Theme Issue 'Mechanisms and functions of brain and behavioural asymmetries'. bottom-up (gene to behaviour) study of this phenomenon (Concha 2004). For example, recent work in the teleost zebrafish has unveiled genetic mechanisms that control the development of neuroanatomical asymmetries (reviewed in Halpern et al. 2003; Concha 2004) and established the first operational links between genetics, asymmetric morphology and lateralized behaviours (Barth et al. 2005).

One of the best-studied cases of brain asymmetry is observed in the epithalamus of vertebrates (Concha $\&$ Wilson 2001; Bianco \& Wilson 2009). In zebrafish, epithalamic asymmetry is established through a sequence of developmental modules. Initially, asymmetry (structural differences between left and right sides at the individual level) and laterality (directionality of asymmetry at a population level) are determined by the coordinated activity of members of the fibroblast growth factor (J. Regan, M. Concha, M. Roussigne, C. Russell and S. Wilson 2007, unpublished data) and nodal (Concha et al. 2000) signalling pathways, respectively. Then, a sequential programme of asymmetric morphogenesis generates neuroanatomical asymmetries in the epithalamic pineal complex and 

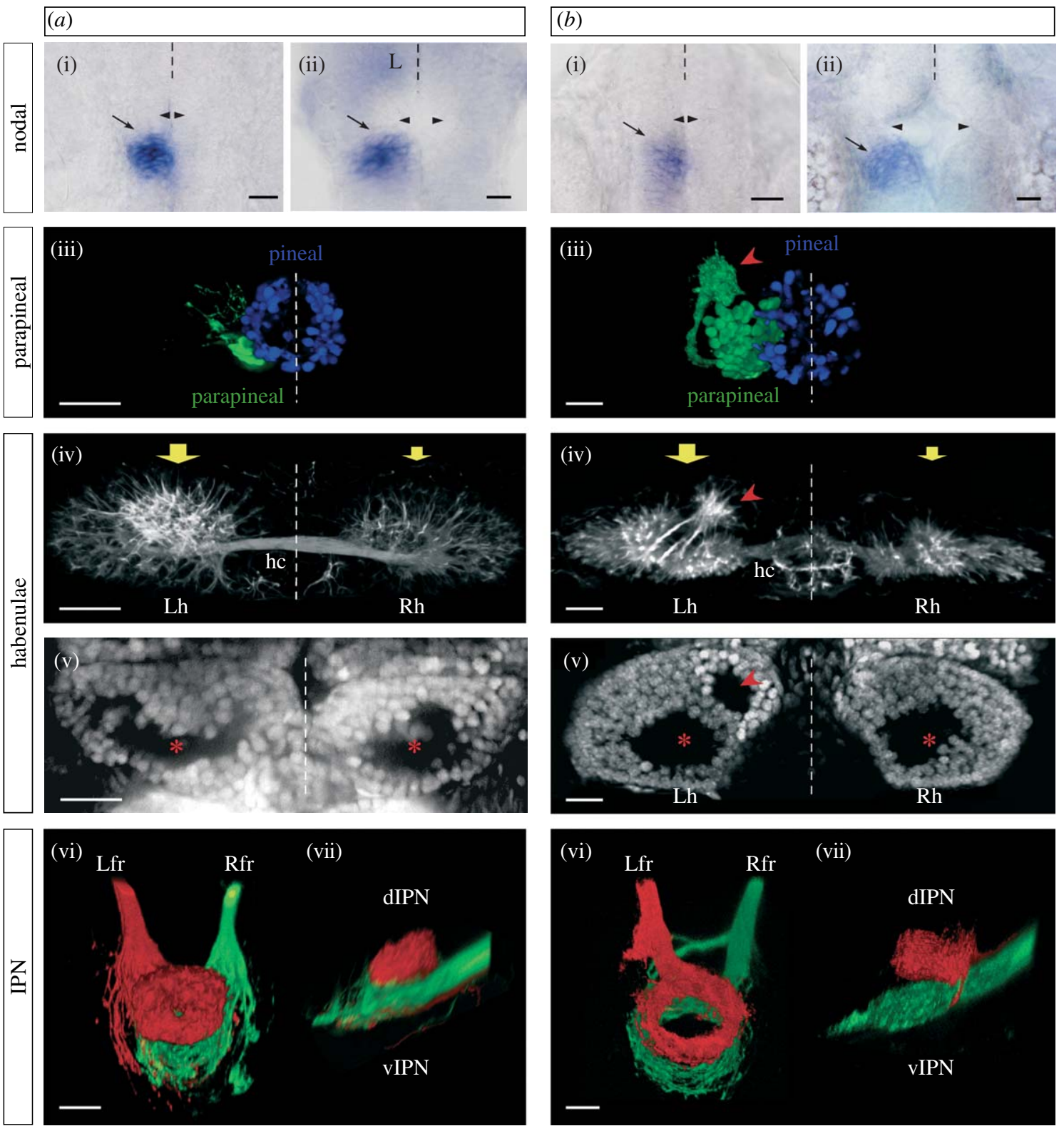

Figure 1. (a) Zebrafish and (b) medaka share an overall pattern of molecular and morphological epithalamic asymmetry. ((i),(ii)) Asymmetric expression of components of the nodal signalling pathway in the presumptive epithalamus. mRNA expression of orthologue genes was detected by whole-mount in situ hybridization (arrows) at (i) normalized STU 35 (Dr-lefty1, Ol-lefty) and (ii) 43 (Dr-pitx2c, Ol-pitx2c) (table 2). The lateral flexure of the third ventricle is indicated by arrowheads. (iii) Left-sided positioning and efferent projection of the parapineal organ. Expression of GFP was detected in medaka $\operatorname{Tg}(f R \times 2:: G F P)$ and zebrafish $\operatorname{Tg}(F o x D 3:: G F P)$ and pseudo-coloured in blue and green to label pineal and parapineal organs, respectively. The red arrowhead points to the terminal dandelion seed-head-shaped domain of parapineal efferent connectivity. (iv) Asymmetric organization of neuropil in the habenulae. Arrows indicate the regions of neuropil, which exhibit dissimilar growth between the left and right habenulae, as detected by immunostaining against acetylated $\alpha$-tubulin. The red arrowhead points to a neuropil domain that is detected exclusively in the left habenula of medaka. (v) Asymmetric organization of neuronal cell bodies in the habenulae. Asterisks indicate equivalent regions of the left and right habenulae. The red arrowhead points to a domain devoid of fluorescent Nissl staining that is detected exclusively in the left habenula of medaka. ((vi),(vii)) Dorsoventral segregation of left-right habenular efferents in the IPN. Left and right habenular projections were labelled with $\mathrm{DiD}$ (red) and $\mathrm{DiO}$ (green), respectively. Images correspond to ((i)-(vi)) dorsal and (vii) lateral views with anterior and dorsal to the top, respectively. Stages of development correspond to $120 \mathrm{HPF}$ (zebrafish, at $26^{\circ} \mathrm{C}$ ) and Iwamatsu's stage 39 (medaka), unless otherwise stated. ((iii)-(vii)) Three-dimensional projections from confocal z-stacks. L, left; R, right; Lh, left habenula; Rh, right habenula; hc, habenular commissure; Lfr, left fasciculus retroflexus; Rfr, right fasciculus retroflexus; dIPN, dorsal domain of the IPN; vIPN, ventral domain of the IPN. Scale bars, ((i) $-(\mathrm{v})) 20 \mu \mathrm{m}$, ((vi),(vii)) $30 \mu \mathrm{m}$.

habenulae. Early asymmetries of the pineal complex involve the asymmetric migration of the photoreceptive parapineal organ to the left side (Concha et al. 2003). Subsequent habenular asymmetries are characterized by differential growth of sub-nuclei (Aizawa et al. 2005,
2007; Gamse et al. 2005) and neuropil domains (Concha et al. 2000) between the left and right sides. Finally, asymmetries in the ratios of different subtypes of habenular projection neurons result in asymmetric target connectivity wherein left and right habenular 

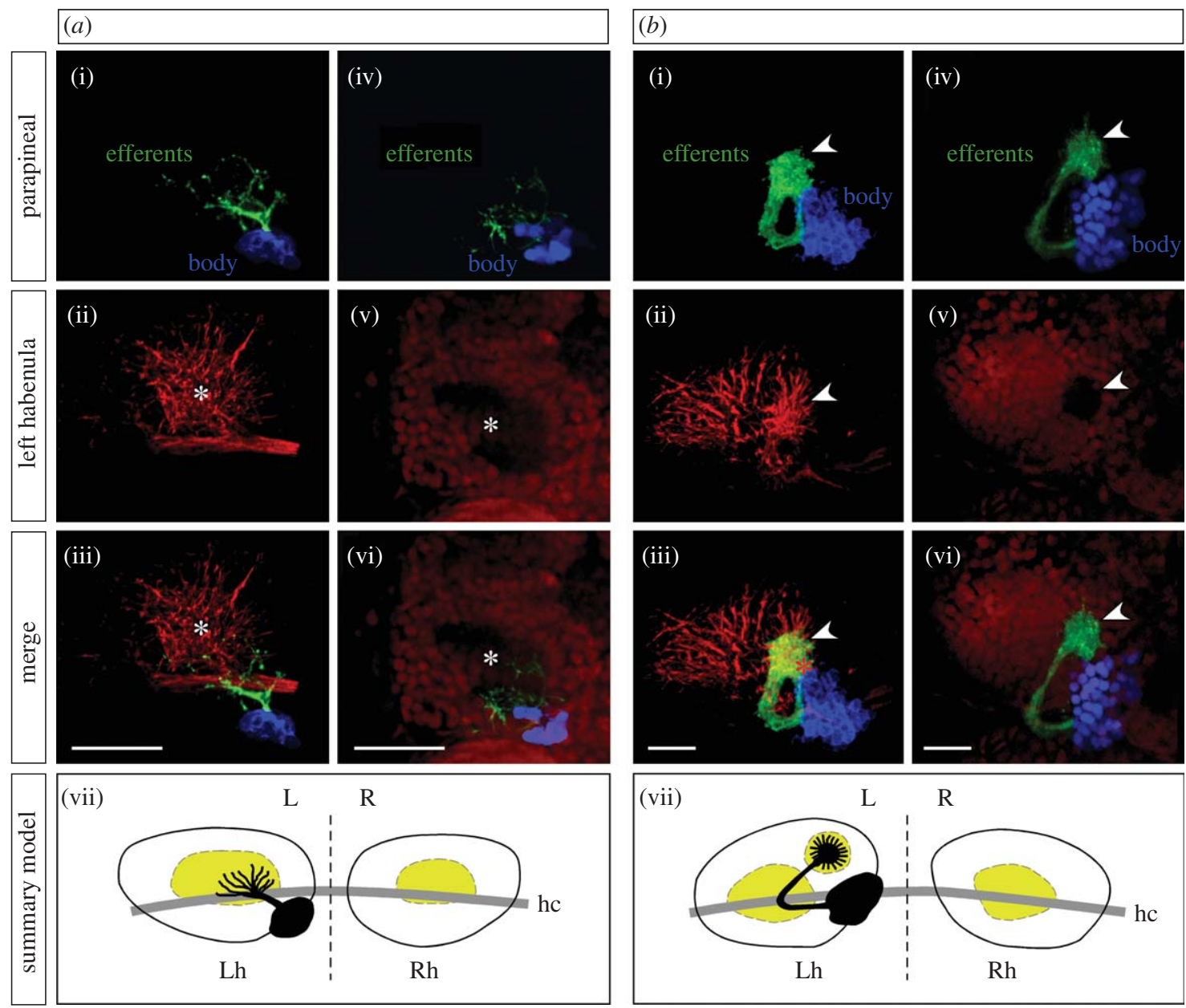

Figure 2. Heterotopic parapineal efferent connectivity in the left habenula of (a) zebrafish and (b) medaka. (a(i)-(vi)) Parapineal efferents blend into a dorsomedial neuropil domain of the left habenula in zebrafish whereas in (b(i)-(vi)) they segregate from other sources of habenular neuropil to form a distinct dorso-anteromedial domain in the left habenula of medaka. Images correspond to dorsal views of the left habenula with anterior to the top. Images were obtained after three-dimensional maximum projections from confocal $z$-stacks. The parapineal organ was pseudo-coloured in blue (parapineal body) and green (parapineal efferents) after immunostaining against GFP in (a(i), (iv)) 120 HPF zebrafish $T g(F o x D 3:: G F P)$ and (b(i),(iv)) St.39 medaka $\operatorname{Tg}(f R \times 2: \because G F P)$. Distribution of neuropil and nuclei in the left habenula were detected by (ii) immunostaining against acetylated $\alpha$-tubulin and (v) fluorescent To-pro staining, respectively. Merged images of double labelling are shown in the bottom panels ((iii),(vi)). Asterisks indicate nuclei-free domains of the left habenula where parapineal connectivity is distributed in zebrafish. Arrowheads point to the terminal dandelion seed-head-shaped domain of parapineal efferent connectivity in medaka. (vii) Summary model of parapineal efferent connectivity in zebrafish and medaka. (a(vii)) In zebrafish, parapineal efferents distribute broadly within a large dorsomedial neuropil domain of the left habenula situated immediately anterior to the habenular commissure. ( $b($ vii)) In medaka, parapineal efferents form a thick bundle of axons, which after entering the left habenula, make a turn towards the midline to end in a well-defined dandelion seed-head-shaped neuropil domain situated in the most dorso-anteromedial aspect of the left habenula. All images correspond to dorsal views, with anterior to the top. The body of the parapineal organ and its efferent connectivity are shown in black, the habenular commissure in grey and neuropil domains in yellow. L, left; R, right; Lh, left habenula; Rh, right habenula; hc, habenular commissure. Scale bars, $20 \mu \mathrm{m}$.

efferents are segregated along the dorsoventral axis of the interpeduncular nucleus (IPN) in the ventral midbrain (Aizawa et al. 2005; Gamse et al. 2005; Bianco et al. 2008).

Three main aspects are important to highlight about the development of epithalamic asymmetries. First, genetic pathways that establish asymmetry are autonomous from those that control laterality (Concha et al. 2000). Such independence in the developmental control of asymmetry and laterality makes the epithalamus of zebrafish an attractive vertebrate model to study the ontogenic (genetic and epigenetic) mechanisms that underlie directional asymmetries, in which most individuals are asymmetrical in the same direction within the population (Van Valen 1962). Second, laterality of epithalamic asymmetry is coupled to laterality of visceral asymmetry (Concha et al. 2000; Long et al. 2003; Carl et al. 2007) in contrast to other structural and functional asymmetries of the vertebrate brain, e.g. asymmetries associated to speech and handedness (Torgersen 1950; Kennedy et al. 1999; Tanaka et al. 1999). This indicates that asymmetries controlled by independent mechanisms coexist in the vertebrate brain. Finally, epithalamic asymmetries are immersed in an evolutionarily conserved circuit involved in limbic-system-related responses (Sutherland 1982; Klemm 2004; Bianco \& Wilson 2009), which has been implicated in the origin of neuropsychiatric disorders 
(Sandyk 1991; Ellison 1994). Altogether, these observations underscore the relevance of understanding the evolutionary origin, genetic control, circuit configuration and behavioural correlates of epithalamic asymmetry to begin dissecting general principles of directional asymmetries and the specific role of the epithalamus, and its associated asymmetric circuit in normality and pathology.

Recent comparative surveys have revealed a striking conservation of epithalamic asymmetry among a wide range of vertebrate species (Concha \& Wilson 2001; Guglielmotti \& Cristino 2006). However, the lack of systematic comparative analyses addressing the genetic and developmental bases hampers the examination of general principles of epithalamic asymmetry development. In this context, the emergence of zebrafish and medaka as complementary model organisms suitable for comparative developmental approaches (FurutaniSeiki \& Wittbrodt 2004) offers a unique opportunity. As lineages of zebrafish (Danio rerio, Order Cypriniformes) and medaka (Oryzias latipes, Order Beloniformes) diverged 115-200 Myr ago, comparison has the potential to unveil those aspects that represent the backbone of epithalamic asymmetry and those that are subjected to evolutionary variation.

In this study, we carried out a first systematic interspecies comparison of brain asymmetry development in teleosts. We analysed the morpho-topological organization of epithalamic asymmetry and studied the temporal organization of developmental modules using a novel method for time normalization based on the rate of somitogenesis. We found a strikingly conserved overall pattern of asymmetry in the parapineal-habenular-interpeduncular system. In spite of this, we observed heterotopic changes in the organization of parapineal efferent connectivity, heterochronic shifts in the timing of developmental events underlying the establishment of asymmetry and divergent degrees of population-level laterality. Altogether, these findings highlight the usefulness of zebrafish and medaka as comparative tools to study the developmental mechanisms of epithalamic asymmetry in vertebrates.

\section{MATERIAL AND METHODS}

\section{(a) Fish lines}

Zebrafish (D. rerio) lines used in this work were wild-type Tübingen and $\operatorname{Tg}($ foxD3::GFP) (Gilmour et al. 2002). Medaka (O. latipes) lines were wild-type Cab, $\operatorname{Tg}(f R \times 2: \because G F P)$ and $T g(f R \times 2 / D E:: G F P)$ (Wittbrodt et al. 2002). Embryos and fry were obtained by natural spawning, raised at $26-28^{\circ} \mathrm{C}$ in standard embryo medium, and staged according to morphology (Kimmel et al. 1995; Iwamatsu 2004) and age (hours post fertilization, HPF).

(b) Whole-mount in situ hybridization, immunohistochemistry, fuorescent Nissl and To-Pro staining

In situ hybridization was performed according to standard protocols for medaka (Loosli et al. 1998) and zebrafish (Westerfield 1996). RNA antisense probes targeted Dr-lefty1 (Bisgrove et al. 1999; Thisse \& Thisse 1999), Dr-pitx2c (Essner et al. 2000), Ol-lefty (Soroldoni et al. 2007) and Ol-pitx2 (Jaszczyszyn et al. 2007). Tubulin immunostaining was performed in larvae fixed in 2 per cent TCA/PBS at room temperature for 1.5 (medaka) and 3 (zebrafish) hours, as described earlier (Concha et al. 2003). Mouse anti-acetylated $\alpha$-tubulin (Sigma, 1:1000), rabbit-anti-green fluorescent protein (GFP) (Abcam, $1: 1000$ ) and Alexa-488/647 conjugated secondary (Molecular Probes, $1: 200$ ) antibodies were used. Fluorescent Nissl staining comprised an overnight incubation with NeuroTrace 530/615 red Nissl (Molecular Probes, $1: 200)$ at $4^{\circ} \mathrm{C}$. Incubation with To-Pro-3 iodide stain (642/661) (Molecular Probes, $1: 1000)$ for 1 hour was used for nuclear counterstaining. Embryos were mounted in glycerol for microscopic observation and photography.

\section{(c) Labelling of habenular efferent projections}

For the labelling of habenular projections, larvae and embryos were immersed in fixative ( $4 \% \mathrm{PFA} / \mathrm{PBS}$ ) and the skin covering the dorsal diencephalon and eyes removed. Crystals of lipophilic dyes $\mathrm{DiD}$ and $\mathrm{DiO}$ (Molecular Probes) were applied in left and right habenulae using tungsten needles connected to a micromanipulator (Aizawa et al. 2005). Labelled larvae were incubated in 0.5 per cent $\mathrm{PFA} / \mathrm{PBS}$ at $4^{\circ} \mathrm{C}$ for 2 days in darkness, to allow lipophilic dyes reach the IPN.

\section{(d) Image acquisition, processing and three-dimensional reconstruction}

Fluorescent samples were imaged on either Zeiss LSM 5 Pascal confocal or UltraView RS spinning disc (Perkin Elmer) microscopes using an Achroplan $40 \times / 0.8 \mathrm{~W}$ dipping objective or a Plan-Apochromat $40 \times / 1.2 \mathrm{~W}$ objective. Images were deconvolved to reduce blurring and noise using Huygens Professional and Scripting Deconvolution softwares. Threedimensional image projections were obtained using the opacity reconstruction model in VoLOCITY software (Improvision).

\section{(e) Rationale and methodology for normalization of developmental time}

According to a hypothetical model of developmental time control, the overall rate of embryo development depends on both intrinsic clock and temperature-sensitive mass-specific metabolic rate signals (Reiss 2003). Zebrafish and medaka exhibit similar size of embryos, larvae and adults and probably share comparable mass-specific metabolic rates. To avoid the influence of temperature upon this variable, we considered the timing of onset and offset of developmental events at a single constant temperature $\left(26^{\circ} \mathrm{C}\right)$. In zebrafish, developmental events were determined as $\mathrm{HPF}$ at $28^{\circ} \mathrm{C}$ and then scaled to $\mathrm{HPF}$ at $26^{\circ} \mathrm{C}$ according to Kimmel et al. (1995). In medaka, timings of developmental events were expressed as HPF at $26^{\circ} \mathrm{C}$ using the Iwamatsu developmental stage table (Iwamatsu 2004). To scale the influence of the internal clock, we normalized absolute times based on the rate of somitogenesis. This periodic segmentation process is known to be controlled by a molecular clock linked to oscillatory gene expression (Saga \& Takeda 2001; Freitas et al. 2005) that depends on the rates of transcription and translation (Giudicelli \& Lewis 2004). We considered the time needed for making a single somite during the linear phase of somitogenesis as a time-normalizing factor, and expressed the newly calculated normalized times in somite time units (STU). The calculation method used available data on the rate of somitogenesis at $26^{\circ} \mathrm{C}$ in zebrafish (Kimmel et al. 1995) and medaka (Iwamatsu 2004). Somite number versus time was plotted using OriginPro v. 7.0220. The linear phase of somitogenesis extended between 4 and 30 somites for both species, and the total number of somites formed was 34 and 35 for zebrafish and medaka, respectively. Linear regression of the data revealed that zebrafish and medaka form 1.7 and 0.797 somites per hour, respectively. The reciprocal of the slope 
values indicated the time needed for making a single somite $(t-1$ som $)$ in both species. Normalized times of development were obtained by dividing absolute time by $t-1$ som.

\section{RESULTS}

\section{(a) Morphological and topological organization of epithalamic asymmetries}

(i) Asymmetric expression of nodal signalling genes in the embryonic epithalamus

In zebrafish, several components of the nodal signalling pathway are asymmetrically expressed in the epithalamus preceding the onset of asymmetric morphogenesis (Concha et al. 2000; Liang et al. 2000). For example, the nodal inhibitor lefty1 (Dr-lefty1) and the downstream transcriptional effector pitx2c (Dr-pitx2c) define restricted dorsal domains of expression in the left side of the neural tube, posterior to the lateral flexure of the diencephalic ventricle (figure $1 a(\mathrm{i})$,(ii)). Recent reports in medaka have shown that Ol-lefty (Carl et al. 2007; Soroldoni et al. 2007) and Ol-pitx2 (Jaszczyszyn et al. 2007) also display asymmetric expression in the dorsal diencephalon, and a close examination indicates that the extent and topology of expression of these genes are similar to zebrafish (compare figure $1 a(\mathrm{i}), b(\mathrm{i})$ and $a$ (ii), $b$ (ii)).

(ii) Left-sided asymmetric positioning and connectivity of the embryonic parapineal organ

In zebrafish, asymmetric morphogenesis of the parapineal organ involves an initial phase of migration from the dorsal midline to the left side of the brain followed by the development of efferent connectivity directed to the left habenula (Concha et al. 2003). Confocal imaging of transgenic $\operatorname{Tg}($ fox $D 3:: G F P)$ zebrafish embryos reveals that the parapineal organ is located on the left and ventral sides of the pineal organ, and sends axonal projections that distribute broadly in the left habenula (figure $1 a$ (iii)). In medaka, the parapineal organ of $\operatorname{Tg}(f R \times 2: \because G F P)$ embryos is also observed on the left side and develops efferent connectivity directed to the left habenula (figure $1 b$ (iii)). However, the volume of the parapineal organ compared with the pineal organ is considerably larger in medaka (ratio of $0.6 / 1 \pm 0.13, n=3$ embryos, mean \pm s.d.) than zebrafish (ratio of $0.1 / 1 \pm 0.02, n=3$ ) (compare figure $1 a$ (iii), $b($ iii)). In addition, parapineal efferents form a thick and long bundle of axons that make an orthogonal turn towards the anterior, dorsal and the midline to end in a well-defined neuropil domain with the shape of a dandelion seed head (figure $1 b$ (iii)).

\section{(iii) Asymmetric cytoarchitectonic organization of the larval habenulae}

In zebrafish, left and right habenular nuclei undergo distinct patterns of neurogenesis (Aizawa et al. 2007) and display asymmetric growth of neuropil domains (Concha et al. 2003; Gamse et al. 2003). We performed immunostaining against acetylated $\alpha$-tubulin to reveal the distribution of neuropil (figure $1 a(\mathrm{iv})$ ) and fluorescent-Nissl staining to expose the spatial organization of neuronal cell bodies (figure $1 a(\mathrm{v})$ ). We confirmed that neuropil asymmetries in zebrafish are limited to a dorsomedial region of the left habenula located in the vicinity of the habenular commissure (arrows in figure $1 a($ iv)). In medaka, neuropil asymmetries define a compact and Nissl well-delimited neuropil domain situated in the most dorso-anteromedial aspect of the left habenula (arrowheads in figure $1 b$ (iv), (v)). Such a singular domain is not observed in the right habenula of medaka and is absent from both left and right habenulae of zebrafish (figure $1 a(\mathrm{iv}),(\mathrm{v}), b(\mathrm{iv}),(\mathrm{v}))$.

\section{(iv) Contribution of parapineal connectivity to habenular asymmetry}

Double immunostaining against acetylated $\alpha$-tubulin (neuropil) and GFP (parapineal organ) in transgenic embryos reveals that parapineal efferents make a hidden morphological contribution to habenular asymmetry in zebrafish. Parapineal efferent connectivity blends into a neuropil domain situated immediately anterior to the habenular commissure, which becomes asymmetrically enlarged in the left habenula compared with the right counterpart (asterisks in figure 2a(i)-(vii); Concha et al. 2003). By contrast, parapineal efferents make a more explicit contribution to morphological asymmetry in medaka. Most parapineal axonal terminals segregate from other sources of habenular neuropil to form a distinct dorso-anteromedial domain situated distant from the habenular commissure, which corresponds to the singular left-sided habenular neuropil domain defined by acetylated $\alpha$-tubulin and Nissl staining (arrowheads in figure 2b(i)-(vii); see also arrowheads in figure $1 b$ (iv),(v)).

\section{(v) Dorsoventral segregation of left-right habenular efferents} in the larval midbrain

The target regions of habenular neurons can be determined by labelling left and right habenular nuclei with the lipophilic dyes $\mathrm{DiD}$ and $\mathrm{DiO}$, respectively (Aizawa et al. 2005). In zebrafish larvae, efferent connectivity from left and right habenular nuclei forms distinct and segregated ring-shaped domains within dorsal and ventral regions of the IPN, respectively (figure 1a(vi),(vii); Aizawa et al. 2005; Gamse et al. 2005; Bianco et al. 2008). A similar pattern of habenular efferent connectivity is observed in the larval IPN of medaka (see figure 1b (vi),(vii); Carl et al. 2007). However, the cross-sectional area of the central fibre-free region of IPN rings, compared with the crosssectional area of the entire IPN appears relatively larger in medaka $(26.7 \pm 3 \%$ of total IPN, $n=3$ embryos, mean \pm s.d.) than in zebrafish $(8.8 \pm 4.6 \%$ of total IPN, $n=3$ ).

\section{(vi) Laterality of epithalamic asymmetries and its} correspondence to organ laterality

In zebrafish, the development of parapineal and habenular asymmetries is interdependent and result in larvae showing concordant laterality of epithalamic asymmetries (Concha et al. 2003; Gamse et al. 2003). In addition, laterality of epithalamic and visceral asymmetries are coupled as both depend on left-sided nodal signalling emerging from a common symmetrybreaking event (Concha 2004; Levin 2005). In medaka, we scored the laterality of parapineal (GFP), 
Table 1. Concordant laterality of epithalamic and heart asymmetries in zebrafish and medaka.

\begin{tabular}{|c|c|c|c|c|c|c|}
\hline \multirow[b]{2}{*}{ parapineal laterality } & \multicolumn{3}{|c|}{ habenular laterality $^{\mathrm{a}}$} & \multicolumn{3}{|c|}{ heart jog/loop laterality ${ }^{\mathrm{b}}$} \\
\hline & left $(\%)$ & right $(\%)$ & no. & normal $(\%)$ & reversed $(\%)$ & no. \\
\hline \multicolumn{7}{|l|}{ zebrafish (Danio rerio) } \\
\hline left & 100 & 0 & 48 & 98 & 0 & 306 \\
\hline right & 0 & 0 & 0 & 0 & 2 & 6 \\
\hline total & 100 & 0 & 48 & 98 & 2 & 312 \\
\hline \multicolumn{7}{|c|}{ medaka (Oryzias latipes) } \\
\hline left & 100 & 0 & 98 & 100 & 0 & 470 \\
\hline right & 0 & 0 & 0 & 0 & 0 & 0 \\
\hline total & 100 & 0 & 98 & 100 & 0 & 470 \\
\hline
\end{tabular}

${ }^{a}$ Concordant laterality of parapineal and habenular asymmetries was analysed by immunostaining against acetylated $\alpha$-tubulin (habenulae) and GFP (parapineal) in $T g\left(\right.$ Foxd3::GFP) (zebrafish, $140 \mathrm{HPF}$ at $26^{\circ} \mathrm{C}$ ) and $\operatorname{Tg}\left(f R \times 2:: G F P\right.$ ) (medaka Iwamatsu St.39, $216 \mathrm{HPF}$ at $26^{\circ} \mathrm{C}$ ).

${ }^{\mathrm{b}}$ Concordant laterality of epithalamic and heart asymmetries was analysed in living embryos by scoring the orientation of heart jog/looping, and the position of the parapineal organ in $T g\left(F o x d 3:: G F P\right.$ ) (zebrafish, $56 \mathrm{HPF}$ at $\left.26^{\circ} \mathrm{C}\right), \operatorname{Tg}(f R \times 2:: G F P$ ) and $\operatorname{Tg}(f R \times 2 / D E:: G F P)$ (medaka, Iwamatsu St.27, $58 \mathrm{HPF}$ at $26^{\circ} \mathrm{C}$ ).

habenular (acetylated $\alpha$-tubulin) and organ (heart looping) asymmetries and found a similar concordant laterality pattern (table 1). Surprisingly, we were unable to find a single case of reversal from normal heart laterality in three different strains of medaka $(T g(f R \times 2::$ GFP $), n=317$ embryos; $T g(f R x 2 / D E:: G F P), n=153$; and cab, $n=148)$, a situation that is common ( $5 \%$ of individuals on average) in other teleost species (Palmer 2004).

\section{(b) Temporal analysis of epithalamic asymmetry development}

To analyse how developmental time of epithalamic asymmetry has changed during the evolution of zebrafish and medaka lineages, we compared three main aspects: sequence (temporal arrangement of developmental modules); relative timing (onset/offset of developmental events with respect to some intrinsic time scale); and duration (overall rate of development). We found that all main developmental modules of epithalamic asymmetry were present in both species and temporally arranged in a similar sequential manner. For example, asymmetric nodal expression preceded left-sided positioning of the parapineal organ, which in turn was followed by the establishment of habenular asymmetry and segregation of habenular efferents in the IPN (figure $3 a$ ).

To perform a meaningful comparison of relative timing and duration, we scaled the absolute time of onset/offset of homologous developmental events to the duration of a conserved periodic process that depends on intrinsic embryo dynamics (e.g. somitogenesis), and produced a normalized time scale that could be compared among taxa (figure $3 b$ and table 2 ; see rationale and description of methodology in \$2). Comparison of normalized developmental times between zebrafish and medaka uncovered three main groups of events that reveal unexpected similarities/ differences in the relative timing. A first group comprised early embryonic processes whose timing of onset became highly coordinated in both species after time normalization. Within this group, we found epiboly, gastrulation (shield formation), onset of expression of hatching enzymes genes (Inohaya et al. 1995, 1997) and somitogenesis (figure 3b). A second group included developmental events whose absolute differences in timing become inverted after time normalization. Important examples within this group were the onset of asymmetric epithalamic nodal signalling, onset of parapineal axonal projection and the initiation of habenula-IPN connectivity (figure $3 b$ ). The onset of asymmetric epithalamic nodal expression exhibited a delay of approximately 6 STU towards later developmental times after normalization, when compared with medaka (table 2; figure $3 b$ ). Interestingly, the magnitude of this delay was comparable with the delay in the onset of heart beating (13 STU) but was considerably smaller than the temporal shift in the initiation of both parapineal axonal projection (50 STU) and habenula-IPN connectivity (40 STU; figure $3 b$ ). Finally, a third group included developmental events whose differences in timing were conserved after time normalization. The single example of this group corresponded to hatching, which occurred at an earlier developmental time in zebrafish than medaka (figure $3 b$ ).

A last step of comparison concerned the duration of developmental events. We focused our analysis on the expression of nodal signalling genes, as they were transient and could be determined with accuracy. Absolute duration of expression of Ol-lefty and Ol-pitx2 doubled that of Dr-lefty 1 and Dr-pitx2c, respectively (table 2; figure $3 a$ ). However, the ratio between the lengths of lefty and pitx2 expressions was equivalent in both species (zebrafish $=0.25$; medaka $=0.24$ ) suggesting that the differences in the absolute length of gene expression could result from variations in the intrinsic speed of embryo development. To test this hypothesis, we compared normalized lengths of gene expression and found them strikingly similar for each pair of orthologue genes: differences represented less than 15 per cent for lefty and 10 per cent for pitx 2 when calculating the ratio zebrafish/medaka (figure $3 b$ ).

\section{DISCUSSION}

\section{(a) Overall conservation of asymmetry in the parapineal-habenular-IPN system of teleosts}

In this study, we compared the main developmental modules of epithalamic asymmetry in two related teleost species with $115-200 \mathrm{Myr}$ of independent 
(a)

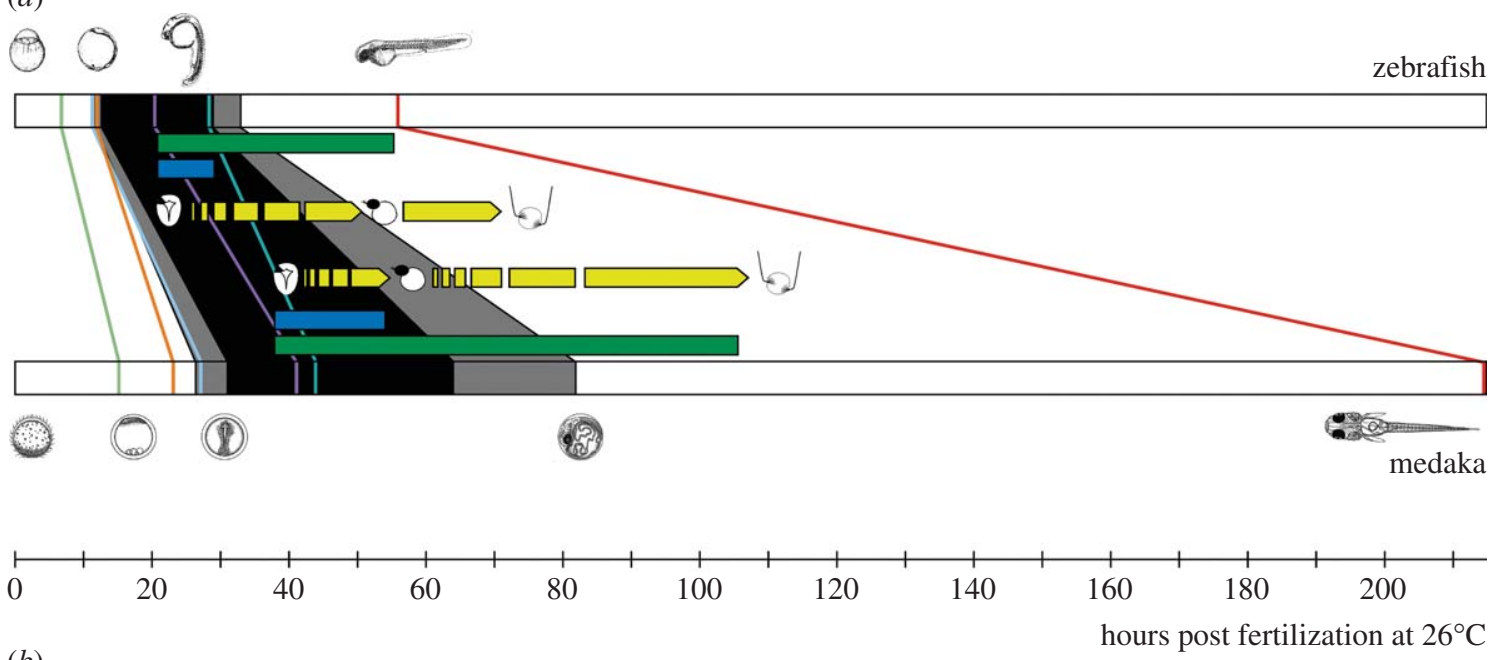

(b)

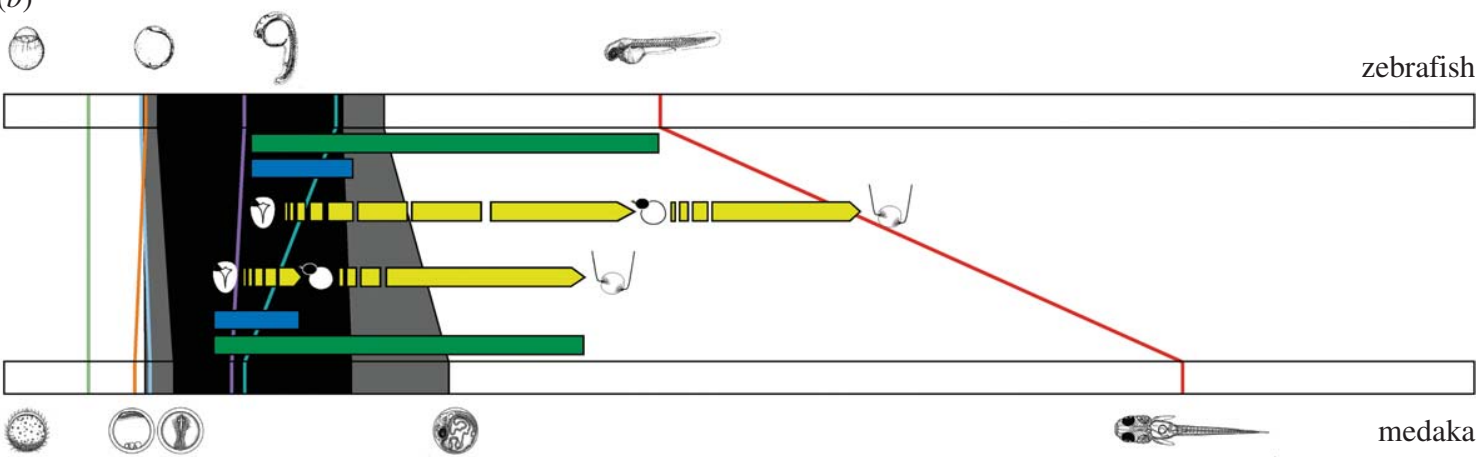

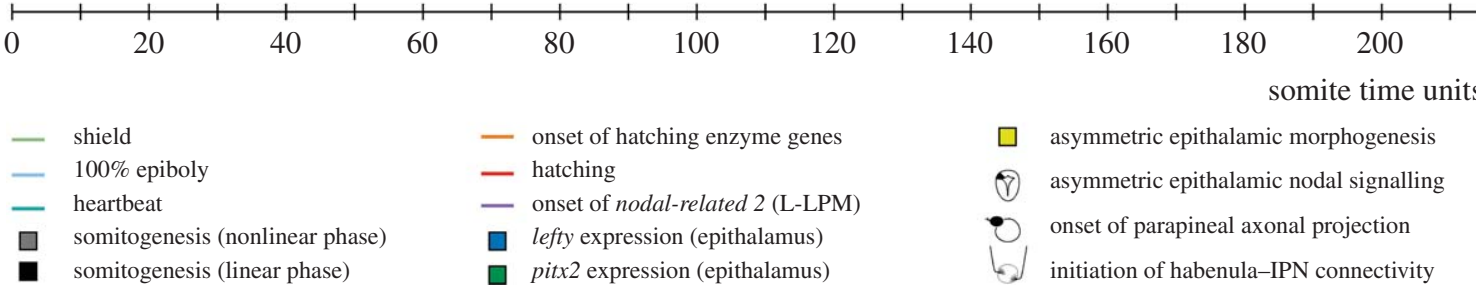

Figure 3. Comparison of sequence, relative timing and duration of developmental events during the establishment of epithalamic asymmetry in zebrafish and medaka. The diagrams show the temporal occurrence of key steps of asymmetric brain morphogenesis in zebrafish and medaka, expressed in $(a)$ absolute and $(b)$ normalized times. To provide a contextual view, the timing of main embryonic events is also included. The colour codes shown at the bottom of the figure indicate different developmental events (lines) and periods (boxes or bars) analysed in the temporal plots of $(a, b)$. For clarity, equivalent events in medaka and zebrafish are joined. Diagrams of developmental stages were obtained from the literature (Kimmel et al. 1995; Iwamatsu 2004). Schematic of epithalamic asymmetry events (bottom right) correspond, from top to bottom, to: a frontal view of the epithalamus depicting left-sided asymmetric nodal expression, a dorsal view of the pineal complex showing the initiation of left-sided parapineal axonal projection and a dorsal view of the IPN (white circle) revealing habenular efferent connectivity reaching dorsal and ventral regions of the IPN. The scale was maintained in $(a, b)$ to emphasize the effect of time normalization. Zebrafish and medaka show a conserved sequence of developmental events of epithalamic asymmetry although they exhibit distinct relative timing (heterochrony).

evolution. Our findings reveal a striking conservation of both the overall spatial organization of brain asymmetry and the temporal sequential arrangement of developmental modules underlying the formation of the parapineal-habenular-IPN system. Such conservative ontogenetic trajectory suggests a causal dependency between the different asymmetry modules. This idea is supported by recent experimental evidence showing that habenular asymmetry is affected by physical removal of the parapineal organ (Concha et al. 2003; Gamse et al. 2003; Bianco et al. 2008). In addition, segregation of habenular efferents in the IPN depends on the proper development of asymmetry in the habenulae (Aizawa et al. 2005; Gamse et al. 2005; Carl et al. 2007; Kuan et al. 2007; Bianco et al. 2008). Evolutionary conservation also suggests that the overall pattern of asymmetry in the parapineal-habenular-IPN axis is plesiomorphic to teleosts. Indeed, habenular and parapineal asymmetries are described in a number of teleost species (Concha \& Wilson 2001) and recent observations extend these findings to the IPN of the southern flounder (Paralichthys lethostigma; Kuan et al. 2007) and guppy (Poecilia reticulata; A. Villalón \& M. L. Concha 2007, unpublished data). Interestingly, despite 
Table 2. Comparison of developmental events of brain asymmetry between zebrafish and medaka.

\begin{tabular}{|c|c|c|c|c|c|c|}
\hline \multirow[b]{2}{*}{ developmental event } & \multicolumn{3}{|c|}{ zebrafish (Danio rerio) } & \multicolumn{3}{|c|}{ medaka (Oryzias latipes) } \\
\hline & time $(\mathrm{HPF})^{\mathrm{a}}$ & & $\mathrm{STU}^{\mathrm{b}}$ & time $(\mathrm{HPF})^{\mathrm{b}}$ & & $S T U^{b}$ \\
\hline \multicolumn{7}{|c|}{ epithalamic nodal expression } \\
\hline \multicolumn{7}{|c|}{ lefty } \\
\hline onset & $19.8 \pm 1$ & $(17 \pm 1)$ & $34.4 \pm 2$ & $36 \pm 2$ & (St.21-22) & $28.7 \pm 2$ \\
\hline offset & $28.5 \pm 1$ & $(24.5 \pm 1)$ & $49.4 \pm 2$ & $52 \pm 2$ & (St.25-26) & $41.4 \pm 2$ \\
\hline duration & $8.7 \pm 1$ & $(6.5 \pm 1)$ & $13.4 \pm 2$ & $16 \pm 2$ & & $12.7 \pm 2$ \\
\hline \multicolumn{7}{|l|}{ pitx2 } \\
\hline onset & $19.8 \pm 1$ & $(17 \pm 1)$ & $34.4 \pm 2$ & $36 \pm 2$ & (St.21-22) & $28.7 \pm 2$ \\
\hline offset & $54.7 \pm 1$ & $(47 \pm 1)$ & $94.4 \pm 2$ & $103.5 \pm 3$ & (St.32-33) & $82.5 \pm 3$ \\
\hline duration & $34.9 \pm 1$ & $(21.5 \pm 1)$ & $58.4 \pm 2$ & $67.5 \pm 3$ & & $53.8 \pm 3$ \\
\hline \multicolumn{7}{|l|}{ parapineal asymmetry } \\
\hline $\begin{array}{l}\text { onset of axonal } \\
\text { projection }\end{array}$ & $57 \pm 1$ & $(49 \pm 1)^{\mathrm{c}}$ & $98.2 \pm 2$ & $66 \pm 8$ & (St.27-29) & $52.6 \pm 7$ \\
\hline \multicolumn{7}{|c|}{ habenular efferents in IPN } \\
\hline $\begin{array}{l}\text { initiation of } \\
\text { connectivity }^{\mathrm{d}}\end{array}$ & $64 \pm 6$ & $(74 \pm 7)$ & $128 \pm 12$ & $111 \pm 10$ & (St.32-33) & $88.5 \pm 8$ \\
\hline
\end{tabular}

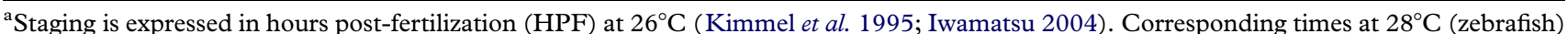
and Iwamatsu stages (St) (medaka) are indicated in brackets. Timing of onset/offset was calculated as the midpoint between the stage when the developmental event is first observed and the preceding/following stage, respectively. Variability corresponds to half the duration of the interval between these stages.

${ }^{\mathrm{b}}$ Normalized times are expressed in STU (see $\S 2$ ).

${ }^{\mathrm{c}}$ Taken from Concha et al. $(2000,2003)$.

${ }^{\mathrm{d}}$ Initiation of connectivity between the habenula and IPN is defined by the initial axonal branching of left and right fasciculi retroflexus within the IPN, prior to the establishment of dorsal and ventral ring-shaped domains.

the overall conservation of habenular asymmetry among a wide range of vertebrate groups (Concha \& Wilson 2001) the segregation of left-right habenular efferents along the dorsoventral axis of the IPN appears unique to teleosts as it is absent in frogs (Rana clamitans), salamanders (Ambystoma maculatum) and mice (Kuan et al. 2007). Whether or not this peculiar asymmetry trait represents a variation of form evolved exclusively by the teleost lineage will need further experimental testing.

\section{(b) Heterotopic parapineal efferent connectivity suggests divergent principles of development between zebrafish and medaka}

Our results support the notion that left-sided positioning of the parapineal organ is a shared feature of asymmetric brain morphogenesis within the teleost group (Borg et al. 1983; Concha \& Wilson 2001). However, the relative size of the parapineal organ (compared with the pineal organ) and its pattern of efferent connectivity greatly differ between zebrafish and medaka. In zebrafish, the body of the parapineal organ is relatively small in size $( \pm 10 \%$ of the pineal) and its efferent connectivity distribute broadly in the left habenula. By contrast, the parapineal organ of medaka is larger ( $\pm 60 \%$ of the pineal) and its efferent connectivity forms a large and well-defined anterodorsomedial neuropil domain within the left habenula (figure 2). Although the number and nature of parapineal-habenular synapses remains unknown, our results suggest that divergent principles of development and circuit configuration emerged during the independent evolution of zebrafish and medaka lineages. Such a variation in the relative size of pineal and parapineal organs is not exclusive to teleosts as it is also observed among species of reptiles developing a parietal eye (the homologous structure to the parapineal organ; Concha \& Wilson 2001).

Previous results suggest that the spatial organization of parapineal efferents depends on a bidirectional interaction established between the parapineal organ and habenulae during development (figure 4). Initially, early asymmetry in the presumptive habenular region is thought to guide asymmetric parapineal migration (Concha et al. 2003). Subsequent left-sided positioning of the parapineal organ is required for the amplification (and perhaps the topological setting) of distinct differentiation programmes in the left and right habenulae (Gamse et al. 2003; Bianco et al. 2008). Finally, parapineal axons distribute in regions of the left habenula, which exhibit enlarged neuropil (Concha et al. 2003) and asymmetric leftover expression (Gamse et al. 2003), therefore linking the topology of parapineal efferent connectivity to the underlying organization of differentiation domains within the left habenula.

Based on these observations, we propose two developmental models to explain the different topologies of parapineal efferent connectivity observed in zebrafish and medaka (figure 4). In the first model, the molecular/connectional identity of parapineal target cells is conserved in the two species, but the topological organization has diverged owing to changes in the spatial and/or temporal organization of a shared set of signals that pattern the habenulae (model 1; figure $4 b$ ). In the second model, the molecular/connectional identity of parapineal target cells has diverged as a result of divergent signalling mechanisms involved in either guiding parapineal connectivity or patterning the habenulae (model 2; figure 4c).

The proposed models have potential dissimilar implications in the function of the parapinealhabenular-IPN system. Whereas solitary changes in 
the topology of parapineal target cells probably represent no major functional modification of the system (model 1; figure 4b), transformations in the identity of parapineal target cells might result in distinct neurotransmitter and/or connectional influences of the habenulae upon the IPN (model 2; figure $4 c$ ). Given the overall morphological and connectional conservation of the parapineal-habenular-IPN circuit, it seems reasonable to expect a conservation of parapineal functionality in the circuit (model 1). Nevertheless, it is possible that the parapineal organ plays no major role in this asymmetric circuit and that the observed phenotypic variation in the topology of parapineal efferent connectivity is a direct consequence of this feature (Hallgrímsson 2003). To date, we have no sufficient data to either sustain or discard this possibility. As the parapineal organ contains both photoneuroendocrine cells and projection neurons, it is possible that circadian variations of light influence the neuroendocrine activity of the parapineal organ and consequently the function of the habenular-IPN system (Concha \& Wilson 2001). However, it has also been reported that parapineal photoreceptors are rather rudimentary (Rudeberg 1969; Van Veen 1982; Ekstrom et al. 1983) and that in many species the parapineal organ appears to have regressed in adulthood (Borg et al. 1983).

\section{(c) Heterochronic shifts and the ontogeny of epithalamic asymmetry}

The dimension of time is critical for development and a key factor in the generation of evolutionary diversity (Gould 1977). The examination of the temporal dimension of development among species allows the study of developmental trajectories, the detection of heterochronies (shifts in timing), the making of inferences about the coupling/uncoupling of developmental modules and the reconstruction of the ancestral sequence of developmental events (Reiss 2003; Zelditch 2003). In the present study, we searched for events of conservation and variation in each of the three main aspects of time underlying the development of epithalamic asymmetries. We found a major conservation in the sequence of developmental modules of brain asymmetry (see above). For a proper examination of relative timing and duration, we developed a method to normalize the intrinsic time scale of zebrafish and medaka development based on the clock properties of somitogenesis. Using this normalization method, we could synchronize the relative timing of early embryonic events. In addition, we found that duration of the expression of genes involved in the control of brain laterality matched after time normalization. This finding provides support to the usefulness of this normalization method for the comparison of developmental time among related species, compared with that of alternative methods (Dettlaff \& Dettlaff 1961; Reiss 1989; Chipman et al. 2000; Clancy et al. 2001). Moreover, this observation suggests that both species share a similar tempo of nodal-dependent laterality determination, and that absolute differences in the duration of nodal signalling depend primarily on the intrinsic rate of embryo development of each species.

The normalization method also allowed the distinction of interspecies changes in the relative timing of epithalamic asymmetry events. Three main heterochronic shifts involved the onset of epithalamic nodal signalling, the onset of parapineal axonal efferent projection and the initiation of habenula-IPN connectivity expressed as the initial branching of left and right axons emerging from the fasciculus retroflexus within the IPN (figure 3). The direction of these shifts is consistent with previous reports suggesting that brain development is delayed relative to somitogenesis in zebrafish compared with medaka (Wittbrodt et al. 2002). More recent data add extra support to this general concept as it reveals a reversal in the relative timing of expression of specific components of the nodal signalling pathway in the brain with respect to the lateral plate mesoderm (LPM) in the two species, e.g. in medaka mRNA of nodal-related 2, lefty and pitx2 are detected earlier in the brain than that in the LPM while the opposite is observed in zebrafish (figure $3 b$; Rebagliati et al. 1998; Bisgrove et al. 2000; Soroldoni et al. 2007). Unexpectedly, the onset of parapineal axonal projection and the initiation of habenula-IPN connectivity exhibited a pronounced heterochronic shift with respect to the onset of nodal signalling, being largely delayed in zebrafish with respect to medaka (figure $3 b$ ). As parapineal connectivity appears to be linked to the programme of habenular differentiation, it is possible that the latter is delayed in zebrafish, and that the more dispersed distribution of parapineal target cells of the zebrafish larvae represents a transitional state towards a more segregated distribution reached in the habenulae at post-larval stages. Consistent with the idea of a shift in the timing of habenular differentiation, we observed that the onset of axonal branching of habenular efferents within the IPN is also delayed in zebrafish compared with medaka (figure $3 b$ ). Further experimental testing of this hypothesis might provide a causal link between the heterotopic and heterochronic changes described in this study.

It is important to note that aspects of organogenesis such as the onset of heart beating are shifted in the same temporal direction as shifts in brain development. This observation opens the possibility that organogenesis as a whole has undergone a heterochronic shift during the evolution of medaka and zebrafish lineages. In this respect, it is intriguing that hatching shows a reversed heterochronic shift to that observed for organogenesis, e.g. it is delayed in medaka compared with zebrafish. As the onset of expression of hatching enzyme genes is comparable in zebrafish and medaka (figure 3b; Inohaya et al. 1995, 1997), it is likely that the differences in hatching time are a result of dissimilar chorion composition and thickness between the two species (Hart et al. 1984; Hart \& Donovan 2005). Regardless of the underlying developmental mechanism, a main consequence of the heterochronic shift in hatching is the definition of zebrafish as altricial (immature) and medaka as precocial (more developed) species (MacArthur \& Wilson 1967).

\section{(d) Is the laterality of asymmetry canalized in medaka?}

Although left-sided laterality of heart asymmetry is a well-conserved trait of vertebrates, a small percentage of individuals in the population show spontaneous 
habenulae $\rightarrow$ parapineal

(a)

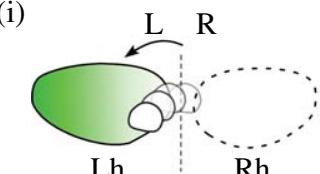

(b)

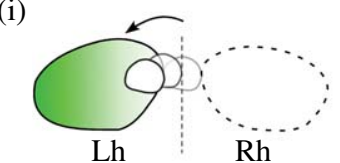

parapineal positioning? habenular pre-pattern?

(c)

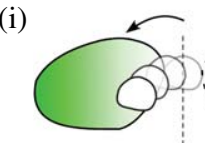

$\mathrm{Lh}$

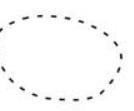

$\mathrm{Rh}$ habenulae $\leftarrow$ parapineal

(ii)

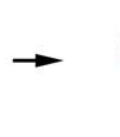

(ii)

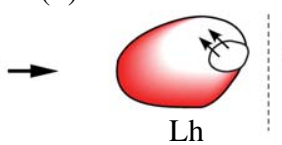

different organization of subdomains equal identity of parapineal target

(ii)
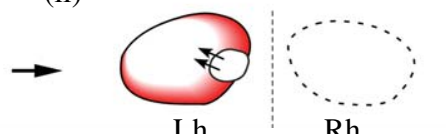

$\mathrm{Rh}$ equal organization of subdomains change in identity of parapineal target habenulae $\rightarrow$ parapineal

(iii)

L $\quad \mathrm{R}$

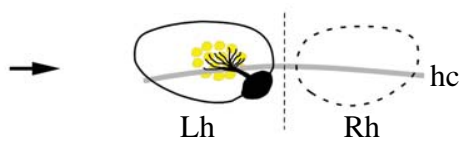

(iii)

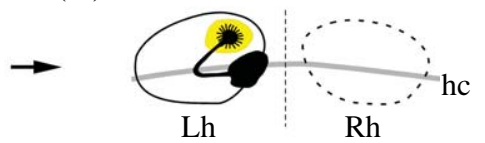

projection to region of equal identity but distinct topology

(iii)

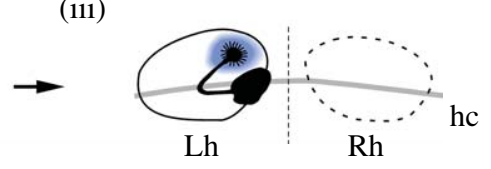

projection to region of distinct identity and topology

Figure 4. Developmental models of heterotopic parapineal efferent connectivity. (a) Zebrafish: asymmetries of the parapineal organ and habenulae interact in three consecutive steps during development. (i) Initial left-right biases in the presumptive habenular region guide parapineal migration to the left side. (ii) Subsequent left-sided positioning of the parapineal organ is involved in the induction/propagation of a distinct spatial pattern of habenular differentiation. (iii) Finally, the topology of subdomains arising after habenular differentiation determines the position of habenular neurons receiving parapineal efferent connectivity. (b) Medaka (model 1): heterotopic parapineal efferent connectivity arises from a different topological organization of habenular subdomains. In this model, spatial differences in the location of the parapineal organ at the time of habenular differentiation and/or underlying differences in habenular (i) pre-patterning lead to (ii) distinct topological programmes of habenular subdomain differentiation and (iii) subsequent positioning of parapineal target cells. (c) Medaka (model 2): heterotopic parapineal efferent connectivity arises from different selection of parapineal target cells within the habenulae. In this model, parapineal migration and habenular differentiation are equivalent in both species. However, parapineal projections reach different target neurons in the habenula of both species owing to the differences in axon guidance cues. (i) Shaded green regions depict molecular left-right biases within the presumptive habenulae. The movement of the parapineal organ from the midline to the left side (arrows) is represented as partially overlapping drawings of parapineal outlines. (ii) White and red regions illustrate putative subdomains of the habenulae. Arrows illustrate the direction of the inductive properties of the parapineal organ. (iii) The topological pattern of parapineal efferent connectivity (black) and the location of parapineal target cells within the habenula (colours) are shown (see also figure 2). Colours represent equivalent cellular identities. All diagrams correspond to dorsal views, with anterior to the top. For clarity, only the left habenula is illustrated, and the right habenula is depicted with dotted lines. L, left; R, right; Lh, left habenula; Rh, right habenula; hc, habenular commissure.

reversal of this asymmetry. Incidence of heart reversals have declined during vertebrate evolution from fishes (approx. 5\%) through amphibians and birds (1-2\%) to mammals (less than $0.1 \%$ ), indicating a canalization of heart laterality during vertebrate evolution (Palmer 2004). Our finding that medaka showed 0 per cent of heart reversals indicates that this species deviates from the expected teleost pattern (e.g. zebrafish, trout and salmon). Although we cannot discard the notion that the inbreeding nature of the medaka strains (Wittbrodt et al. 2002) reduces the normal fluctuation of individual laterality, it is possible that symmetrybreaking mechanisms are more robust and resistant to genetic and environmental perturbations in medaka than in the other teleosts that have been analysed. A major mechanism of vertebrate laterality determination involves the generation of extracellular leftward fluid flow (the so-called nodal flow) within the ventral node of mice (Hirokawa et al. 2006) and the Kupffer's vesicle (KV) of teleosts (Essner et al. 2005; Kramer-Zucker et al. 2005; Okada et al. 2005). Recent reports have revealed that the $\mathrm{KV}$ of medaka shares more similarities to the mammalian node than to the zebrafish $\mathrm{KV}$, when considering the cytoarchitectonic organization of ciliated cells and the robustness of the nodal flow (Essner et al. 2005; Kramer-Zucker et al. 2005; Okada et al. 2005; Hirokawa et al. 2006; Oteiza et al. 2008). Hence, we propose that the canalization of embryo laterality may be linked to the morphology of laterality organs and consequently the nature of the nodal flow they produce. In this context, other developmental conditions that have been proposed to make laterality decisions more predictable (e.g. placental environments; Palmer 2004) would play only additive roles.

\section{CONCLUSIONS: ZEBRAFISH AND MEDAKA AS MODELS FOR COMPARATIVE DEVELOPMENTAL BIOLOGY OF VERTEBRATE BRAIN ASYMMETRY}

Since the initial proposal of medaka and zebrafish as complementary model organisms suitable for comparative developmental biology (Furutani-Seiki \& Wittbrodt 2004), several reports have made use of the experimental and evolutionary advantages of these genetic organisms to start revealing conserved and species-specific principles of vertebrate development 
(e.g. Lynn Lamoreux et al. 2005; Gajewski et al. 2006; Carl et al. 2007). The present study brings additional support to this notion, offers new tools for time comparison between these species and provides novel comparative data and hypotheses to start addressing the ontogenic mechanisms that explain interspecies variations of epithalamic asymmetry. Together, these findings highlight the usefulness of zebrafish and medaka as comparative models of brain asymmetry development and function.

All procedures of animal care and management conformed to high standards in agreement with the revised Council of Europe guidelines (ETS123) on housing, and were approved by a local Committee of Bioethics on Animal Experimentation at the Faculty of Medicine, University of Chile.

We are particularly grateful to Jorge Mpodozis, Gonzalo Marín, Francisco Aboitiz, Stephen Wilson, Isaac Bianco and Mathias Carl for their valuable comments on the versions of the manuscript, and to Dina Silva, Micaela Ricca and Alejandro Chamorro for fish care. Work on the epithalamus and asymmetry in our group is supported by the Howard Hughes Medical Institute (HHMI INTNL 55005940), the Chilean Commission of Science and Technology (PBCT ACT47, BMBF/CONICYT 2003-4-124), the Millennium Scienice Initiative (ICM) and a grant from the European Communities entitled 'Evolution and Development of Cognitive, Behavioural and Neural Lateralisation' (FP62004-NEST-PATH EDCBNL).

\section{REFERENCES}

Aizawa, H., Bianco, I. H., Hamaoka, T., Miyashita, T., Uemura, O., Concha, M. L., Russell, C., Wilson, S. W. \& Okamoto, H. 2005 Laterotopic representation of left-right information onto the dorso-ventral axis of a zebrafish midbrain target nucleus. Curr. Biol. 15, 238-243. (doi:10. 1016/j.cub.2005.01.014)

Aizawa, H., Goto, M., Sato, T. \& Okamoto, H. 2007 Temporally regulated asymmetric neurogenesis causes left-right difference in the zebrafish habenular structures. Dev. Cell 12, 87-98. (doi:10.1016/j.devcel.2006.10.004)

Barth, K. A., Miklosi, A., Watkins, J., Bianco, I. H., Wilson, S. W. \& Andrew, R. J. 2005 fsi zebrafish show concordant reversal of laterality of viscera, neuroanatomy, and a subset of behavioral responses. Curr. Biol. 15, 844-850. (doi:10. 1016/j.cub.2005.03.047)

Bianco, I. H. \& Wilson, S. W. 2009 The habenular nuclei: a conserved asymmetric relay station in the vertebrate brain. Phil. Trans. R. Soc. B 364, 1005-1020. (doi:10.1098/rstb. 2008.0213)

Bianco, I. H., Carl, M., Russell, C., Clarke, J. D. \& Wilson, S. W. 2008 Brain asymmetry is encoded at the level of axon terminal morphology. Neural Dev. 3, 9.

Bisgrove, B. W., Essner, J. J. \& Yost, H. J. 1999 Regulation of midline development by antagonism of lefty and nodal signaling. Development (Camb. Engl.) 126, 3253-3262.

Bisgrove, B. W., Essner, J. J. \& Yost, H. J. 2000 Multiple pathways in the midline regulate concordant brain, heart and gut left-right asymmetry. Development (Camb. Engl.) 127, 3567-3579.

Borg, B., Ekström, P. \& Van Veen, T. 1983 The parapineal organ of teleosts. Acta Zool. (Stockholm) 64, 211-218.

Carl, M., Bianco, I. H., Bajoghli, B., Aghaallaei, N., Czerny, T. \& Wilson, S. W. $2007 \mathrm{Wnt} /$ Axin 1/beta-catenin signaling regulates asymmetric nodal activation, elaboration, and concordance of CNS asymmetries. Neuron 55, 393-405. (doi:10.1016/j.neuron.2007.07.007)
Chipman, A. D., Haas, A., Tchernov, E. \& Khaner, O. 2000 Variation in anuran embryogenesis: differences in sequence and timing of early developmental events. f. Exp. Zool. 288, 352-365. (doi:10.1002/1097-010X (20001215)288:4<352::AID-JEZ8 > 3.0.CO;2-2)

Clancy, B., Darlington, R. B. \& Finlay, B. L. 2001 Translating developmental time across mammalian species. Neuroscience 105, 7-17. (doi:10.1016/S0306-4522(01)00171-3)

Concha, M. L. 2004 The dorsal diencephalic conduction system of zebrafish as a model of vertebrate brain lateralisation. Neuroreport 15, 1843-1846. (doi:10.1097/ 00001756-200408260-00001)

Concha, M. L. \& Wilson, S. W. 2001 Asymmetry in the epithalamus of vertebrates. F. Anat. 199, 63-84. (doi:10. 1017/S0021878201008329)

Concha, M. L., Burdine, R. D., Russell, C., Schier, A. F. \& Wilson, S. W. 2000 A nodal signaling pathway regulates the laterality of neuroanatomical asymmetries in the zebrafish forebrain. Neuron 28, 399-409. (doi:10.1016/ S0896-6273(00)00120-3)

Concha, M. L. et al. 2003 Local tissue interactions across the dorsal midline of the forebrain establish CNS laterality. Neuron 39, 423-438. (doi:10.1016/S0896-6273 (03)00437-9)

Dettlaff, T. A. \& Dettlaff, A. A. 1961 On relative dimensionless characteristics of the development duration in embryology. Arch. Biol. 72, 1-16.

Ekstrom, P., Borg, B. \& Van Veen, T. 1983 Ontogenetic development of the pineal organ, parapineal organ, and retina of the three-spined stickleback, Gasterosteus aculeatus L. (Teleostei). Development of photoreceptors. Cell Tiss. Res. 233, 593-609. (doi:10.1007/BF00212227)

Ellison, G. 1994 Stimulant-induced psychosis, the dopamine theory of schizophrenia, and the habenula. Brain Res. Rev. 19, 223-239. (doi:10.1016/0165-0173(94)90012-4)

Escalante-Mead, P. R., Minshew, N. J. \& Sweeney, J. A. 2003 Abnormal brain lateralization in high-functioning autism. F. Autism Dev. Disord. 33, 539-543. (doi:10.1023/A: 10258 87713788)

Essner, J. J., Branford, W. W., Zhang, J. \& Yost, H. J. 2000 Mesendoderm and left-right brain, heart and gut development are differentially regulated by pitx 2 isoforms. Development (Camb. Engl.) 127, 1081-1093.

Essner, J. J., Amack, J. D., Nyholm, M. K., Harris, E. B. \& Yost, H. J. 2005 Kupffer's vesicle is a ciliated organ of asymmetry in the zebrafish embryo that initiates left-right development of the brain, heart and gut. Development (Camb. Engl.) 132, 1247-1260. (doi:10.1242/dev.01663)

Freitas, C., Rodrigues, S., Saude, L. \& Palmeirim, I. 2005 Running after the clock. Int. F. Dev. Biol. 49, 317-324. (doi:10.1387/ijdb.041955cf)

Furutani-Seiki, M. \& Wittbrodt, J. 2004 Medaka and zebrafish, an evolutionary twin study. Mech. Dev. 121, 629-637. (doi:10.1016/j.mod.2004.05.010)

Gajewski, M., Elmasri, H., Girschick, M., Sieger, D. \& Winkler, C. 2006 Comparative analysis of her genes during fish somitogenesis suggests a mouse/chick-like mode of oscillation in medaka. Dev. Genes Evol. 216, 315-332. (doi:10.1007/s00427-006-0059-6)

Gamse, J. T., Thisse, C., Thisse, B. \& Halpern, M. E. 2003 The parapineal mediates left-right asymmetry in the zebrafish diencephalon. Development (Camb. Engl.) 130, 1059-1068. (doi:10.1242/dev.00270)

Gamse, J. T., Kuan, Y. S., Macurak, M., Brosamle, C., Thisse, B., Thisse, C. \& Halpern, M. E. 2005 Directional asymmetry of the zebrafish epithalamus guides dorsoventral innervation of the midbrain target. Development (Camb. Engl.) 132, 4869-4881. (doi:10.1242/dev.02046) 
Gilmour, D. T., Maischein, H. M. \& Nusslein-Volhard, C. 2002 Migration and function of a glial subtype in the vertebrate peripheral nervous system. Neuron 34, 577-588. (doi:10. 1016/S0896-6273(02)00683-9)

Giudicelli, F. \& Lewis, J. 2004 The vertebrate segmentation clock. Curr. Opin. Genet. Dev. 14, 407-414. (doi:10.1016/ j.gde.2004.06.014)

Gould, S. J. 1977 Ontogeny and phylogeny. Cambridge, MA: Harvard University Press.

Guglielmotti, V. \& Cristino, L. 2006 The interplay between the pineal complex and the habenular nuclei in lower vertebrates in the context of the evolution of cerebral asymmetry. Brain Res. Bull. 69, 475-488. (doi:10.1016/ j.brainresbull.2006.03.010)

Güntürkün, O., Diekamp, B., Manns, M., Nottelmann, F., Prior, H., Schwarz, A. \& Skiba, M. 2000 Asymmetry pays: visual lateralization improves discrimination success in pigeons. Curr. Biol. 10, 1079-1081. (doi:10.1016/S09609822(00)00671-0)

Hallgrímsson, B. 2003 Variation. In Keywords and concepts in evolutionary developmental biology (eds B. K. Hall \& W. M. Olson), pp. 368-377. London, UK: Harvard University Press.

Halpern, M. E., Liang, J. O. \& Gamse, J. T. 2003 Leaning to the left: laterality in the zebrafish forebrain. Trends Neurosci. 26, 308-313. (doi:10.1016/s0166-2236(03) 00129-2)

Hart, N. H. \& Donovan, M. 2005 Fine structure of the chorion and site of sperm entry in the egg of Brachydanio. F. Exp. Zool. 227, 277-296. (doi:10.1002/ jez.1402270212)

Hart, N. H., Pietri, R. \& Donovan, M. 1984 The structure of the chorion and associated surface filaments in Oryzias: evidence for the presence of extracellular tubules. F. Exp. Zool. 230, 273-296. (doi:10.1002/jez. 1402300213)

Hirokawa, N., Tanaka, Y., Okada, Y. \& Takeda, S. 2006 Nodal flow and the generation of left-right asymmetry. Cell 125, 33-45. (doi:10.1016/j.cell.2006.03.002)

Hutsler, J. \& Galuske, R. A. 2003 Hemispheric asymmetries in cerebral cortical networks. Trends Neurosci. 26, 429-435. (doi:10.1016/S0166-2236(03)00198-X)

Inohaya, K., Yasumasu, S., Ishimaru, M., Ohyama, A., Iuchi, I. \& Yamagami, K. 1995 Temporal and spatial patterns of gene expression for the hatching enzyme in the teleost embryo, Oryzias latipes. Dev. Biol. 171, 374-385. (doi:10. 1006/dbio.1995.1289)

Inohaya, K., Yasumasu, S., Araki, K., Naruse, K., Yamazaki, K., Yasumasu, I., Iuchi, I. \& Yamagami, K. 1997 Speciesdependent migration of fish hatching gland cells that express astacin-like proteases in common [corrected]. Dev. Growth Differ. 39, 191-197. (doi:10.1046/j.1440169X.1997.t01-1-00007.x)

Iwamatsu, T. 2004 Stages of normal development in the medaka Oryzias latipes. Mech. Dev. 121, 605-618. (doi:10. 1016/j.mod.2004.03.012)

Jaszczyszyn, Y., Haeussler, M., Heuze, A., DebiaisThibaud, M., Casane, D., Bourrat, F. \& Joly, J. S. 2007 Comparison of the expression of medaka (Oryzias latipes) pitx genes with other vertebrates shows high conservation and a case of functional shuffling in the pituitary. Gene 406, 42-50. (doi:10.1016/j.gene.2007. 05.024)

Kennedy, D. N., O’Craven, K. M., Ticho, B. S., Goldstein, A. M., Makris, N. \& Henson, J. W. 1999 Structural and functional brain asymmetries in human situs inversus totalis. Neurology 53, 1260-1265.
Kimmel, C. B., Ballard, W. W., Kimmel, S. R., Ullmann, B. \& Schilling, T. F. 1995 Stages of embryonic development of the zebrafish. Dev. Dyn. 203, 253-310. (doi:10.1002/ aja.1002030302)

Klemm, W. R. 2004 Habenular and interpeduncularis nuclei: shared components in multiple-function networks. Med. Sci. Monit. 10, RA261-RA273.

Kramer-Zucker, A. G., Olale, F., Haycraft, C. J., Yoder, B. K., Schier, A. F. \& Drummond, I. A. 2005 Cilia-driven fluid flow in the zebrafish pronephros, brain and Kupffer's vesicle is required for normal organogenesis. Development (Camb. Engl.) 132, 1907-1921. (doi:10.1242/dev.01772)

Kuan, Y. S., Gamse, J. T., Schreiber, A. M. \& Halpern, M. E. 2007 Selective asymmetry in a conserved forebrain to midbrain projection. F. Exp. Zool. Part B 308, 669-678. (doi:10.1002/jez.b.21184)

Levin, M. 2005 Left-right asymmetry in embryonic development: a comprehensive review. Mech. Dev. 122, 3-25. (doi:10.1016/j.mod.2004.08.006)

Li, X., Branch, C. A., Ardekani, B. A., Bertisch, H., Hicks, C. \& DeLisi, L. E. 2007 fMRI study of language activation in schizophrenia, schizoaffective disorder and in individuals genetically at high risk. Schizophr. Res. 96, 14-24. (doi:10. 1016/j.schres.2007.07.013)

Liang, J. O., Etheridge, A., Hantsoo, L., Rubinstein, A. L., Nowak, S. J., Izpisua Belmonte, J. C. \& Halpern, M. E. 2000 Asymmetric nodal signaling in the zebrafish diencephalon positions the pineal organ. Development (Camb. Engl.) 127, 5101-5112.

Long, S., Ahmad, N. \& Rebagliati, M. 2003 The zebrafish nodal-related gene southpaw is required for visceral and diencephalic left-right asymmetry. Development (Camb. Engl.) 130, 2303-2316. (doi:10.1242/dev.00436)

Loosli, F., Koster, R. W., Carl, M., Krone, A. \& Wittbrodt, J. 1998 Six3, a medaka homologue of the Drosophila homeobox gene sine oculis is expressed in the anterior embryonic shield and the developing eye. Mech. Dev. 74, 159-164. (doi:10.1016/S0925-4773(98) 00055-0)

Lynn Lamoreux, M., Kelsh, R. N., Wakamatsu, Y. \& Ozato, K. 2005 Pigment pattern formation in the medaka embryo. Pigment Cell Res. 18, 64-73. (doi:10.1111/ j.1600-0749.2005.00216.x)

MacArthur, R. H. \& Wilson, E. O. 1967 The theory of island biogeography. Princeton, NJ: Princeton University Press.

Okada, Y., Takeda, S., Tanaka, Y., Belmonte, J. C. \& Hirokawa, N. 2005 Mechanism of nodal flow: a conserved symmetry breaking event in left-right axis determination. Cell 121, 633-644. (doi:10.1016/j.cell.2005.04.008)

Oteiza, P., Koppen, M., Concha, M. L. \& Heisenberg, C. P. 2008 Origin and shaping of the laterality organ in zebrafish. Development (Camb. Engl.) 135, 2807-2813.

Palmer, A. R. 2004 Symmetry breaking and the evolution of development. Science (New York, NY) 306, 828-833. (doi:10.1126/science.1103707)

Pascual, A., Huang, K. L., Neveu, J. \& Preat, T. 2004 Neuroanatomy: brain asymmetry and long-term memory. Nature 427, 605-606. (doi:10.1038/427605a)

Rebagliati, M. R., Toyama, R., Haffter, P. \& Dawid, I. B. 1998 cyclops encodes a nodal-related factor involved in midline signaling. Proc. Natl Acad. Sci. USA 95, 9932-9937. (doi:10.1073/pnas.95.17.9932)

Reiss, J. O. 1989 The meaning of developmental time: a metric for comparative embryology. Am. Nat. 134, 170-189. (doi:10.1086/284974)

Reiss, J. O. 2003 Time. In Keywords and concepts in evolutionary developmental biology (eds B. K. Hall \& W. M. Olson), pp. 358-368. London, UK: Harvard University Press. 
Rogers, L. J. 2000 Evolution of hemispheric specialization: advantages and disadvantages. Brain Lang. 73, 236-253. (doi:10.1006/brln.2000.2305)

Rogers, L. J. \& Andrew, R. J. 2002 Comparative vertebrate lateralization. Cambridge, UK: Cambridge University Press.

Rogers, L. J. \& Vallortigara, G. 2008 From antenna to antenna: lateral shift of olfactory memory recall by honeybees. PLoS ONE 3, e2340. (doi:10.1371/journal. pone.0002340)

Rudeberg, C. 1969 Structure of the parapineal organ of the adult rainbow trout, Salmo gairdneri Richardson. Zeitschrift fur Zellforschung 93, 282-304. (doi:10.1007/ BF00336695)

Saga, Y. \& Takeda, H. 2001 The making of the somite: molecular events in vertebrate segmentation. Nat. Rev. 2, $835-845$.

Sandyk, R. 1991 Relevance of the habenular complex to neuropsychiatry: a review and hypothesis. Int. F. Neurosci. 61, 189-219.

Sherman, G. F., Galaburda, A. M. \& Geschwind, N. 1982 Neuroanatomical asymmetries in non-human species. Trends Neurosci. 5, 429-431. (doi:10.1016/0166-2236 (82)90233-8)

Soroldoni, D., Bajoghli, B., Aghaallaei, N. \& Czerny, T. 2007 Dynamic expression pattern of nodal-related genes during left-right development in medaka. Gene Exp. Patt. 7, 93-101. (doi:10.1016/j.modgep.2006.05.013)

Sutherland, R. J. 1982 The dorsal diencephalic conduction system: a review of the anotomy and functions of the habenular complex. Neurosci. Biobehav. Rev. 6, 1-13. (doi:10.1016/0149-7634(82)90003-3)

Tanaka, S., Kanzaki, R., Yoshibayashi, M., Kamiya, T. \& Sugishita, M. 1999 Dichotic listening in patients with situs inversus: brain asymmetry and situs asymmetry. Neuropsychologia 37, 869-874. (doi:10.1016/S0028-3932(98) 00144-4)

Thisse, C. \& Thisse, B. 1999 Antivin, a novel and divergent member of the TGFbeta superfamily, negatively regulates mesoderm induction. Development (Camb. Engl.) 126, 229-240.

Toga, A. W. \& Thompson, P. M. 2003 Mapping brain asymmetry. Nat. Rev. Neurosci. 4, 37-48. (doi:10.1038/ nrn1009)

Torgersen, J. 1950 Situs inversus, asymmetry, and twinning. Am. F. Hum. Genet. 2, 361-370.

Toth, C., Rajput, M. \& Rajput, A. H. 2004 Anomalies of asymmetry of clinical signs in Parkinsonism. Mov. Disord. 19, 151-157. (doi:10.1002/mds.10685)

Vallortigara, G. \& Rogers, L. J. 2005 Survival with an asymmetrical brain: advantages and disadvantages of cerebral lateralization. Behav. Brain Sci. 28, 575-589, discussion 589-633. (doi:10.1017/s0140525x05000105)

Van Valen, L. 1962 A study of fluctuating asymmetry. Evolution 16, 125-142. (doi:10.2307/2406192)

Van Veen, T. 1982 The parapineal and pineal organs of the elver (glass eel), Anguilla anguilla L. Cell Tiss. Res. 222, 433-444. (doi:10.1007/BF00213223)

Westerfield, M. 1996 The zebrafish book. Oregon, OR: University of Oregon Press.

Wittbrodt, J., Shima, A. \& Schartl, M. 2002 Medaka: a model organism from the Far East. Nat. Rev. 3, 53-64. (doi:10.1038/nrg704)

Zelditch, M. 2003 Space, time and repatterning. In Keywords and concepts in evolutionary developmental biology (eds B. K. Hall \& W. M. Olson), pp. 341-349. London, UK: Harvard University Press. 